\title{
eHealth Spare Parts as a Service: Modular eHealth Solutions and Medical Device Reform
}

\author{
Nadezhda Purtova \\ Assistant professor, Tilburg Institute for Law, Technology, and Society (TILT), \\ Tilburg University, The Netherlands
}

\begin{abstract}
eHealth Platform as a Service ('PaaS') is an innovative way to build mHealth apps out of cloud-based generic components. Having examined the current and future regimes of safety and performance, this article concludes that the 'selling features' of the PaaS (outsourced creation and maintenance of cloud-based parts for easy mHealthbuilding) undermine legal compliance, and the reform will not change this significantly. Although no safety and performance requirements apply yet to consumer eHealth, the medical apps are regulated. Their manufacturers must ensure the final apps are safe, while not being in control of the parts composing the apps. Generic components not meant for a specific medical device and their providers are not subject to safety and performance requirements. Market will likely push PaaS providers to offer mHealth manufacturers tools to ensure and control safety and performance when medical apps are concerned. I foresee no such incentives for consumer mHealth.
\end{abstract}

\section{Keywords}

eHealth - mHealth - EU medical device reform - cloud computing - modular eHealth - safety and performance of health apps

\footnotetext{
* The research for this article was conducted within the project 'Future Internet: socialtechnological alignment in healthcare' (FI-STAR) funded by the European Union's Seventh Programme for research, technological development and demonstration under grant agreement No 604691, online at https://www.fi-star.eu.
} 
eHealth $^{1}$ has firmly entered our daily lives: multiple health-, wellbeing and lifestyle mobile applications helps us manage our physical activity, calorie intake, weight loss or get into a healthy sleeping pattern. The sector of professional medical care, especially so in public healthcare, will too likely embrace eHealth to relief the tension between limited budgets and the rising costs of treatments; growing complexity and costs of care for an ageing population; and increasing expectations and demand for higher care quality. ${ }^{2} \mathrm{~A}$ quick glance at the level of investment in eHealth-related innovation suggests eHealth is here to stay. ${ }^{3}$

A recent development in the field of eHealth is eHealth Platform as a Service ('eHealth PaaS'). eHealth PaaS is a cloud service model meant to simplify the development of distributed mHealth applications by providing app developers with ready-to-use purpose-oriented components with a generic functionality. These generic elements can be used to build unique and different mHealth applications. ${ }^{4}$

This article addresses two eHealth-related innovations: one technological (the eHealth PaaS), and one regulatory. On a technological side, although mHealth applications and the applicable legal requirements have been a subject of academic analysis, ${ }^{5}$ how these requirements apply to the eHealth

1 European Commission defines eHealth as 'the use of ICT in health products, services and processes combined with organisational change in healthcare systems and new skills, in order to improve health of citizens, efficiency and productivity in healthcare delivery, and the economic and social value of health'. (European Commission, 2012. 'Communication from the European Commission to the European Parliament, the Council, the European Economic and Social Committee and the Committee of the Regions - e-health action plan 2012-2020 - Innovative healthcare for the 21st Century', available online at http://ec.europa .eu/health/ehealth/docs/com_2012_736_en.pdf).

2 Deloitte Centre for Health Solutions 'Connected health. How digital technology is transforming health and social care', (Deloitte, London, 2014), p. 6.

3 See an overview of EU-funded eHealth projects at https:/ec.europa.eu/digital-agenda/ en/node/76661\#Factsheets (retrieved 8 May 2015); see also European Commission Communication, 2010, 'A strategy for smart, sustainable and inclusive growth', Сом (2010)2020 final, Brussels, 3.3.2010, available at http://eur-lex.europa.eu/LexUriServ/LexUriServ.do?uri =COM:2010:2020:FIN:EN:PDF (accessed 10 May 2015).

4 For a full catalogue of the FI-STAR components ('enablers') see http://catalogue.fi-star.eu/ enablers.

5 E.g., P. Quinn, A.-K. Habbig, E. Mantovani and P. De Hert, 'The Data Protection and Medical Device Frameworks - Obstacles to the Deployment of mHealth across Europe?', European Journal of Health Law 20(2) (2013) 185-204. 
PaaS and to modular approach to building eHealth applications has not been examined. The regulatory innovation this article considers is the EU reform of safety and performance regime for medical devices. The reform passed several benchmarks in the past year: in October 2015 the Council agreed on its General Approach to the proposed Regulation on medical devices (the 'Medical Device Regulation'), and in May 2016 a political agreement was reached on the final text. ${ }^{6}$ The accounts of the latest steps in the reform in the eHealth law literature are scarce. This article aims to fill in this gap and examines how compatible the adoption of eHealth PaaS as a toolkit for creating health apps is with the EU safety and performance requirements to health technology, both under the current and new regimes.

The analysis unfolds as follows. Section 2 introduces eHealth PaaS and the remainder of this article covers the relevant law. The legal analysis follows the taxonomy for descriptive research in law and technology. ${ }^{7}$ The taxonomy is a useful tool to map a regulatory landscape of a novel technology where the potentially relevant regulations still need to be identified and the way these regulations apply to the technological innovation needs to be explicated and assessed. Following the logic of the taxonomy to move from identifying a broader area of law that is potentially relevant down to more specific analysis of how these norms will work in the context of the new technology, Section 3 starts with identifying broadly which legal regimes of safety and performance apply to eHealth and therefore may be of relevance to eHealth PaaS. I review the general EU regime of product safety and a specific regime of safety and performance of medical devices. Section 4 explicates how the regime of safety and performance of medical devices applies in the particular circumstances of eHealth PaaS, namely, whether or not the generic 'spare parts' available via the PaaS are subject to the safety and performance regulation, as opposed to the finished mHealth apps built of those parts. Section 5 briefly reflects on the challenges that the manufacturers of the mHealth apps may encounter when complying with their obligations to ensure that all the parts interacting

6 Council of the European Union, 2015. General Approach to Proposal for a Regulation of the European Parliament and of the Council on medical devices, and amending Directive 2001/83/EC, Regulation (EC) No 178/2002 and Regulation (EC) No 1223/2009, Brussels 21.9.2015, available at http://data.consilium.europa.eu/doc/document/ST-12040-2015-REV-1/ en/pdf; and Consolidated text after the political agreement, available at http://data.consilium .europa.eu/doc/document/ST-9364-2016-REV-3/en/pdf.

7 B.-J. Koops, 'A taxonomy for descriptive research in law and technology', in: E. Palmerini and E. Stradella (eds.), Law and Technology: The Challenge Of Regulating Technological (Pisa: Pisa University Press, 2013) pp. 37-57. 
within the app are compatible and safe and reliable as a whole when those parts are provided for and controlled, under some circumstances, by a third party. Section 6 sums up the findings of the article to support the key point: the main 'selling features' of the eHealth PaaS, namely, outsourced expertise and burden of creating and maintaining cloud-based spare parts for mHealth 'quick fixes' will also present the main obstacles for legal compliance for the manufacturers of the regulated medical apps, and the reform will not change this significantly.

The final caveat is that at the time of writing, the political agreement on the Medical Device Reform is still to be formally endorsed by the European institutions and go through language checks. ${ }^{8}$ Therefore some changes, albeit minor, in the wording and provisions numbering are possible in the officially published text, expected in the end of the year. All references this text will make to the medical device reform will refer to the text of the political agreement reached in May 2016 as the latest publicly available full reform text, unless explicitly stated otherwise.

eHealth PaaS is a cloud service meant to simplify the development of eHealth applications. Generally, eHealth PaaS offers advantages as any cloud computing solution does. Cloud computing refers to a body of web-based - as opposed to on-premises - services, such as providing storage and computing capacity. ${ }^{9}$ Cloud computing is often presented as a more cost-efficient IT solution: instead of maintaining the expensive, complete IT infrastructure that is required for the on-premises execution of relevant information processing, customers of cloud computing vendors pay only for the services they consume, and avoid the need to use on-premises spaces for the hardware, occupy computing capacity with downloadable software, employ unnecessary IT-personnel, etc.

Specifically, and regardless of the context of application, Platform as a Service provides its users with the capability

8 European Council 'Medical devices: deal reached on new Eu rules', Press release published in Brussels, 25.05.2016 available at http://www.consilium.europa.eu/en/press/ press-releases/2016/05/25-medical-devices/.

9 J.R. Martin and N. Hoover, 'Guide to Cloud Computing', Information Week: The Business Value of Technology (2008). 
to deploy onto the cloud infrastructure consumer-created or acquired applications created using programming languages, libraries, services, and tools supported by the provider. The consumer does not manage or control the underlying cloud infrastructure including network, servers, operating systems, or storage, but has control over the deployed applications and possibly configuration settings for the application-hosting environment. ${ }^{10}$

PaaS can change how eHealth is built and operated. To illustrate, one of the goals of FI-STAR, a EU Seventh Framework project, ${ }^{11}$ was to introduce PaaS to the health sector. The idea is that the FI-STAR eHealth PaaS operates as a toolbox for eHealth app developers. It provides app developers with ready to use 'spare parts'. These spare parts, or purpose-oriented components, are comparable to building blocks, each with a generic functionality of varying degrees of sophistication: connectivity, electronic health records support, or health questionnaires, ${ }^{12}$ and potentially complex data analysis and decision support. These ready components can be combined with each other, or with other own or third party tools at the client's disposal, to build a range of unique and different eHealth applications. ${ }^{13}$ The advantage of such cloud service for health is that building eHealth applications becomes easier, quicker and less skill-intensive for the PaaS clients. Without expertise in engineering or investment of time and funds in developing own technology, any hospital, a general practitioner's surgery, a pharmacy or a start-up should be able to build a new and unique eHealth app, by simply using the ready generic components from the eHealth platform. For instance, FI-STAR tested a diabetes diary, a 2-D barcoding solution for real-time reverse medicament supply chain, an app improving access to care for patients with mental health problems, and others. ${ }^{14}$ But there can also be other specialized medical and consumer wellbeing and lifestyle apps built using eHealth PaaS components, anything from a food or sleep diary app to a health and fitness coach, to an artificial-intelligence

10 U.s. Department of Commerce, National Institute of Standards and Technology, 'The NIST definition of cloud computing. Recommendations of NIST', September 2011, 3 (hereinafter: 'The NIST Recommendations').

11 Website of the Future Internet: social-technological alignment in healthcare ('FI-STAR'), online at https://www.fi-star.eu/.

12 Full catalogue of the FI-STAR components ('enablers') in FI-STAR Catalogue, 2013, available at http://catalogue.fi-star.eu/enablers, retrieved 30 October 2015.

13 C. Thuemmler, et al., 'Norms and standards in modular medical architecture', e-Health Networking, Applications \& Services (Healthcom), October 2013, 382-387.

14 For a full list of the use cases see https://www.fi-star.eu/use-cases.html. 
operated 'digital psychologist'. The level of sophistication depends on the sophistication of the available 'spare parts'.

As any cloud service, eHealth PaaS can be offered as a public, community, or private cloud, or a combination of the three: each layer of the cloud service the hardware infrastructure and the platform itself - can be deployed in a different mode.

Table 1 presents an overview of the main characteristics of these deployment modes: who can use a cloud service (availability), who can own, manage and operate the cloud, and the location of the infrastructure with regard to the client's premises.

These characteristics are decisive in the analysis of compatibility of eHealth PaaS with the legal landscape of safety and performance of health technology. The subsequent sections address key legal challenges that could form obstacles for adoption of the eHealth PaaS from a legal compliance point of view.

TABLE $1 \quad$ eHealth PaaS deployment modes ${ }^{15}$

\begin{tabular}{|c|c|c|c|}
\hline & Public Cloud & Community Cloud & Private Cloud \\
\hline Available to & $\begin{array}{l}\text { Available to } \\
\text { general public. }\end{array}$ & $\begin{array}{l}\text { Available to a } \\
\text { restricted community } \\
\text { of users with shared } \\
\text { needs (e.g. a group of } \\
\text { medical app manufac- } \\
\text { turers, hospitals, etc.). }\end{array}$ & $\begin{array}{l}\text { Available to the } \\
\text { exclusive use of a } \\
\text { single organization } \\
\text { (e.g. a hospital). }\end{array}$ \\
\hline $\begin{array}{l}\text { Owned, } \\
\text { managed, } \\
\text { and } \\
\text { operated by }\end{array}$ & $\begin{array}{l}\text { Third party - } \\
\text { PaaS provider. }\end{array}$ & $\begin{array}{l}\text { Third party — PaaS } \\
\text { provider, or the } \\
\text { community. }\end{array}$ & $\begin{array}{l}\text { May be owned, } \\
\text { managed, and } \\
\text { operated by the } \\
\text { organization, a third } \\
\text { party, or some } \\
\text { combination of } \\
\text { them. }\end{array}$ \\
\hline Located & Off premises. & On or off premises. & On or off premises. \\
\hline
\end{tabular}

15 National Institute of Standards and Technology, 'The NIST definition of cloud computing. Recommendations of NIST', published September 2011, 3 (hereinafter: 'The NIST Recommendations'). 

Safety and Performance Regime of mHealth

Safety for users' health and reliable performance in conformity with intended parameters logically are among key factors of trust in and adoption of any new technology in the healthcare sector. mHealth solutions, directly or indirectly, are intended to produce real health effects, which are meant to be positive: improve quality of sleep or coach to adopt better eating or exercise habits. These effects can also be non-existent or negative in case of a technology malfunction. Yet, the applicability of any safety and performance regime to mHealth is still a grey area, ridden with legal uncertainty.

\subsection{Current Legal Status of mHealth Apps, Subjective Intent of the Manufacturer}

The legal status of mHealth apps as a product or a medical device is currently uncertain. Consequently, whether or not any safety and performance regime applies and, if yes, which one, is uncertain as well. The safety and performance of a large body of health apps appears unregulated, i.e. subject to no safety and performance requirements at all.

Safety and performance of products brought on the European market are currently regulated either by Directive $2001 / 95 / \mathrm{EC}^{16}$ on general product safety (General Product Safety Directive, 'GPSD’), or by specialised legislation applicable to a specific kind of products. The specialised legislation directly relevant for eHealth is the Council Directive 93/42/EEC on medical devices ('MDD'). ${ }^{17}$ The GPSD applies when and to the extent the specific legislation is insufficient or absent. Hence, in order to be subject to any legal regime of safety and performance an mHealth app needs to be, legally speaking, either a 'product', or a 'medical device'. These concepts define the material scope of the Directives and are fundamental for establishing which set of the requirements is applicable. Yet, both concepts present difficulties in the context of mHealth.

Article 1(2)(a) MDD defines a medical device as:

16 Directive 2001/95/EC of the European Parliament and the Council of 3 December 2001 on general product safety, Official Journal 11 l11/4, 15.1.2002.

17 MDD is one of the three medical device directives together forming the European Medical Device Framework. The other directives are Council Directive 90/385/EEC on active implantable medical devices ('AIMDD'), and Directive 98/79/EC of the European Parliament and of the Council on in vitro diagnostic medical devices ('IVDD'). 
any instrument, apparatus, appliance, software, material or other article, whether used alone or in combination, including the software intended by its manufacturer to be used specifically for diagnostic and/or therapeutic purposes and necessary for its proper application, intended by the manufacturer to be used for human beings for the purpose of:

- diagnosis, prevention, monitoring, treatment or alleviation of disease,

- diagnosis, monitoring, treatment, alleviation of or compensation for an injury or handicap,

- investigation, replacement or modification of the anatomy or of a physiological process,

- control of conception,

and which does not achieve its principal intended action in or on the human body by pharmacological, immunological or metabolic means, but which may be assisted in its function by such means.

There are two essential elements to this definition: (1) the objective element of a medical function that a device should fulfil; and (2) the subjective element of the manufacturer's intent to produce a device to serve a medical purpose. The apps that do not fall under this definition, often wellbeing and lifestyle apps, are not medical devices and hence, not subject to MDD.

The list of 'medical purposes' which a device must serve is formulated broadly and allows a significant room for interpretation in individual cases. Yet, there are no binding EU rules concerning the delimitation between lifestyle and wellbeing apps (not subject to MDD) and apps that are medical devices (subject to MDD). ${ }^{18}$ In 2012 the European Commission passed Guidelines on the qualification and classification of stand-alone software used in healthcare within the regulatory framework of medical devices. ${ }^{19}$ The Guidelines are meant, among others, to aid manufacturers in deciding if the software they are building is a medical device, by means of a decision tree and examples.

18 European Commission (2014), 'Commission Staff Working Document on existing EU legal framework applicable to lifestyle and wellbeing apps, Accompanying the document Green Paper on Mobile Health ('mHealth')', сом (2014) 219 final, Brussels, 10 April 2014 (hereinafter 'Staff Working Document'), p. 3.

19 European Commission, 'Guidelines on the qualification and classification of standalone software used in healthcare within the regulatory framework of medical devices', MEDDEV 2.1/6 January 2012 ('MEDDEV 2.1/6 January 2012'). 
Bellow follows a short overview of the criteria decisive for the status of a medical device, according to the Guidelines.

The software, to be considered as a medical device, needs to be stand-alone, i.e. not incorporated in an existing medical device. Otherwise, it is considered part of that medical device and is subject to the respective safety and performance requirements. ${ }^{20} \mathrm{Next}$, software must have an advanced function that goes beyond 'simple storage, archival, communication, "simple search" or lossless compression, ${ }^{21}$ e.g. to modify information (e.g. an MRI image) to 'facilitate the perceptual and/or interpretative tasks performed by the healthcare professionals when reviewing medical information'.22 The medical function should also serve the benefit of an individual patient, as opposed to statistical evaluations of clinical or epidemiological studies, and other actions serving collective good. ${ }^{23}$

The Commission completes its Guidelines with examples of apps that are likely to be medical devices. ${ }^{24}$ The examples include Computer Aided Detection systems intended to provide information 'that may suggest or exclude medical conditions', e.g. through automatic reading of x-ray images or interpret ECGs; ${ }^{25}$ modules intended to generate alarms; ${ }^{26}$ decision-support systems 'which combine medical knowledge databases and algorithms with patient specific data. They are intended to provide healthcare professionals and/or users with recommendations for diagnosis, prognosis, monitoring and treatment of individual patients, ${ }^{27}$ and other.

The Guidelines also contain examples of software functionalities that do not lead to qualification as a medical device according to M EDDEv. ${ }^{28}$ However, the rationale to list some solutions as medical and other as non-medical is not always clear. Namely, there are cases where two solutions performing a comparable function (e.g. information transfer) are classified as both medi$\mathrm{cal}$ and non-medical. For instance, modules that provide patient's treatment information to the paramedics in the ambulance to start the patient's treatment while the patient is being transported are considered medical devices. ${ }^{29}$

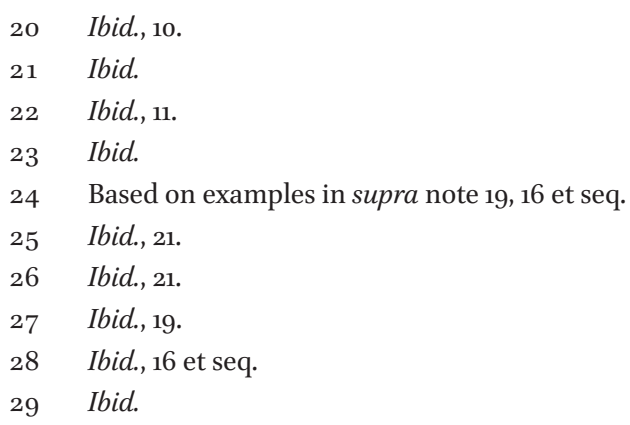


At the same time, other solutions also performing data transfer are not considered medical devices, e.g. a CIS/PDMs - a software based system intended for e.g. intensive care units to store and transfer patient information generated in association with the patient's intensive care treatment (not intended to generate alarm). ${ }^{30}$ The confusion is further exacerbated by the non-binding nature of the MEDDEV guidelines.

A key factor defining a medical device (and its accessory) under MDD, in addition to a medical function, remains to be the manufacturer's intent to have an app (or generally a device) used by medical professionals specifically for one of the medical purposes in Article 1(2)(a) MDD.

The defining role of the manufacturer's intent to design a device specifically for medical use has become a matter of a reference for preliminary ruling in Brain Products GmbH v. BioSemi voF and Others. ${ }^{31}$ The national proceedings before a German court concerned a dispute on whether or not a device called 'ActiveTwo' which enables human brain activity to be recorded is to be classified as a medical device intended for investigation of a physiological process (brain activity), despite the fact that the manufacturer did not intend it for medical use, and hence its marketing without a CE mark (required for medical devices) is to be prohibited. The legal issue was that a medical purpose was not apparent in the third indent of Article 1(2)(a) MDD - the expression 'investigation of a physiological process' - 'unlike in the first and second indents of that provision, where [...] the words 'disease', 'injury', 'handicap' and 'treatment' refer to such a purpose'. ${ }^{32}$ The Court of Justice interpreted the clause in question in light of the context and general objectives of the MDD (as opposed to the literate interpretation). ${ }^{33}$ As regards the context, the Court observed that, among others, the title of the Directive, several references to 'patients', and the Commission Guidelines concerning the definition of a medical device suggest that it is not sufficient for the device to be used in a medical context in order for it to fall under a definition of a medical device. ${ }^{34}$ Rather, a device is 'medical' 'only if the intended purpose of that device, defined by its manufacturer, is medical. ${ }^{35}$ As regards the objectives of the Directive, the MDD provisions must reconcile two core objectives and ensure not only

\footnotetext{
$30 \quad$ Ibid., 21.

31 Judgment of 22 November 2012, Brain Products GmbH v. BioSemi voF and Others, C-219/11, EU:C:2012:742.

32 Judgment in Brain Products GmbH v. BioSemi voF and Others, EU:C:2012:742, paragraph 12.

33 Ibid., paragraph 13.

34 Ibid., paragraphs 14-24.

35 Ibid., paragraph 18.
} 
the protection of patients' health, but also the free movement of medical devices. ${ }^{36}$ The limitations on the freedom of movement of goods as a result of safety and performance requirements associated with a status of a medical device should only be imposed when it is necessary for protection of public health, ${ }^{37}$ and cannot be justified when a product is not 'conceived by its manufacturer to be used for medical purposes'. ${ }^{38}$

Similar to some sports goods which 'enable the functioning of certain organs in the human body to be measured without any medical use' that the Court explicitly rules not be medical devices, ${ }^{39}$ this judgement precludes qualification of eHealth wearables and mHealth apps without specific manufacturer's intent for medical use as medical devices subject to MDD regime, certainly under the 'investigation of a physiological process' clause, and possibly under other clauses. Importantly, the fact that a device not explicitly conceived as medical (but as a lifestyle or wellbeing device, or a device meant for 'domestic' use or even medical research) can be put to a medical use is not sufficient to attribute a medical device status. Hence the manufacturer's explicit intent remains a leading factor.

The intent is to be determined on the basis of 'the data supplied by the manufacturer on the labelling, in the instructions and/or in promotional materials. ${ }^{40}$ A key legal challenge for ensuring the safety and performance of the eHealth technology in general, is that the subjective will of a device manufacturer serves as a trigger for the application of the only safety and performance regime in the area of eHealth, yet, a simple disclaimer stating that the device is not intended to serve medical purposes will release the device manufacturer of any safety and performance obligations. ${ }^{41}$ Advocate General Mengozzi whose opinion the Court followed in Brain Products does not regard possible abuse of this clause as a problem as - 'including for obvious reasons of professional liability' - the consistent practice of medical structures is to purchase only

$36 \quad$ Ibid., paragraphs $25-28$.

37 Ibid., paragraphs 29.

$38 \quad$ Ibid., paragraph 3 o.

39 Judgment in Brain Products GmbH v. BioSemi vOF and Others, EU:C:2012:742, paragraph 31.

40 Article 1(2)(g) of MDD, supra note 19, 11.

41 The Court in Brain Products did not adopt AG Mengozzi's conclusion that '[e]ven if the information provided by the manufacturer is the key factor in determining whether a product is intended to be used for a medical purpose, any product which, by its very nature, is clearly intended to be used solely for a purpose of a medical nature will have to be regarded as a medical device, even if the manufacturer does not describe it as such'. Judgment in Brain Products GmbH v. BioSemi voF and Others, EU:C:2012:742, Opinion of AG Mengozzi, paragraph 63 . 
products which have been certified in accordance with the directive.42 The Advocate General continues that other, often more stringent, regimes of safety exist that would be applicable to products which are not medical devices. ${ }^{43}$ However, software in general and mHealth applications in particular may be an exception and create a specific challenge in this respect as demonstrated further.

The Commission Staff Working Document 'On the existing Eu legal framework applicable to lifestyle and wellbeing apps' explains that it is unclear if and to what extent apps (and presumably other software) that do not qualify as medical devices are subject to GPSD, as the latter 'appli[es] to manufactured products, ${ }^{44}$ and presumably, not software. While the definition of a medical device explicitly includes software, the software is not mentioned in the definition of a product in Article 2(a) GPSD. In addition, lifestyle and wellbeing apps (and other software) may be beyond the scope of GPSD as one of the Directive's goals is 'ensuring a consistent, high level of consumer health and safety protection', ${ }^{45}$ while the Commission Staff Working Document points out that ' $[\mathrm{i}] \mathrm{t}$ is not yet clear if and to what extent lifestyle and wellbeing apps could pose a risk to citizens' health. ${ }^{\prime 6}$ In case a further (e.g., judicial) interpretation of the definition of a product will include software, and the latter will be found to pose safety and health risks, the GPSD may apply to the lifestyle and wellbeing apps that are not medical devices in future. However, at present, the relevance of G PSD for mHealth apps remains unclear. Hence, no legal requirements will be considered in relation to the GPSD.

To conclude, there are two types of mHealth apps: $m$ Health apps specifically intended by a manufacturer for a medical purpose and subject to the MDD safety and performance regime; and mHealth apps that are not intended for a medical purpose, and hence not subject to any safety and performance regime. This gap in regulation does not necessarily present a problem in the context of professional medical care, since the established practice of the medical institutions corrects possible abuses and only devices with certification under the MDD are used. However, this is not the abuse alone that presents a problem. An entire group of mHealth apps exists where apps are genuinely conceived by their manufacturers as lifestyle or wellbeing, for 'domestic' use and hence

\footnotetext{
$42 \quad$ Ibid.

43 Ibid., paragraph 64.

44 Supra note $18,3$.

45 Recital 26 GPSD.

46 Supra note 18, p. 3.
} 
not subject to MDD. These apps often are meant to produce real health effects, and may also create real health risks. Yet, they are not unequivocally subject to the general product or any other safety regulations.

\subsection{Towards 'Objectivisation' of the MDR Regime?}

The uncertain legal status of mHealth solutions and resulting gap in safety regulations have been acknowledged in the Reform efforts. Although the definition of a medical device remained unchanged except for small changes of wording and the inclusion of in vitro diagnostics medical devices, the intent of the Regulation is to include non-medical devices 'similar to medical devices in terms of functioning and risks profile ${ }^{\prime 4}$ in its scope. This signifies a shift towards 'objectivisation' of the MDR regime compared to the MDD in a sense that the application of the safety requirements is triggered by objective risks to health (recognized by the Commission) rather than the subjective intent of the manufacturer alone.

The definition of a medical device under the MDR remains without significant changes. According to Article 2(1)(1) MDR,

'medical device' means any instrument, apparatus, appliance, software, implant, reagent, material or other article, intended by the manufacturer to be used, alone or in combination, for human beings for one or more of the specific medical purposes of:

- diagnosis, prevention, monitoring, prediction, prognosis, treatment or alleviation of disease, diagnosis,

- monitoring, treatment, alleviation of or compensation for an injury or disability,

- investigation, replacement or modification of the anatomy or of a physiological or pathological process or state,

- providing information by means of in vitro examination of specimens derived from the human body, including organ, blood and tissue donations,

and which does not achieve its principal intended action by pharmacological, immunological or metabolic means, in or on the human body, but which may be assisted in its function by such means. 
As it is the case under MDD, the definition has two key components: 1) the objective list of medical purposes that the device should serve, and 2) a subjective intent of the manufacturer.

As for the objective criterion of the medical purposes, these are as generally formulated as under the MDD, which may call for guidelines similar to the current MEDDEV. It is generally up to the Member States to decide on a caseby-case basis whether or not a product falls within the scope of this Regulation (Recital 8 MDR). However, the Commission is empowered, on request of the Member States or independently, and after consulting an expert authority the Medical Device Coordination Group (MDCG), to determine whether a specific product or a group of products falls under a definition of a medical device (Article $3 \mathrm{MDR}$ ). The improvement compared to the current regime is that such Commission guidelines will be binding and will reduce the uncertainty in applying the definition.

The second key element defining a medical device remains to be a subjective intent of the manufacturer. Consequently, the future MDR regime may suffer from the same vulnerability as the current MDD, i.e. a disclaimer by a manufacturer will suffice to exclude the device from the scope of the safety and performance requirements.

Simultaneously, some caveats in the definition may be read as pointing to the 'objectivisation' of the definition of a medical device. Similarly to the MDD, 'intended purpose' is to be observed on the basis of 'the data supplied by the manufacturer on the label, in the instructions for use or in promotional or sales materials or statements and as specified by the manufacturer in the clinical evaluation' ${ }^{48}$ The wording here differs slightly from the current formulation and includes statements of the manufacturer, also made in the course of the clinical evaluation. This change, even if slightly, opens up a possibility to argue that a device is subject to the MDR even though the documentation states otherwise, if the device is marketed, including oral statements, to medical professionals as intended for a medical purpose. This change will likely eliminate the possibility to abuse the 'intent' criterion to avoid compliance.

The final reform text did not adopt the Parliament amendment to the definition of a medical device where the device had to be intended by the manufacturer for one or more 'specific direct or indirect medical purposes'49 (original

48 Article 2(1)(10) MDR.

49 European Parliament legislative resolution of 2 April 2014 on the proposal for a regulation of the European Parliament and of the Council on medical devices, and amending Directive 2001/83/EC, Regulation (EC) No 178/2002 and Regulation (EC) No 1223/2009 $\left(\operatorname{COM}(2012) 0542-\mathrm{C}_{7-0318 / 2012}-2012 / 0266(\mathrm{COD})\right)$ available at: http://www.euro 
emphasis). The reference to 'direct or indirect medical purposes', depending on interpretation, could have helped further 'objectivisation' of the definition by allowing to argue that although the manufacturer did not intend a medical purpose directly, the purpose declared as intended is in fact a medical purpose indirectly, e.g. because it supports medical decision-making and therapy (e.g. a diabetes diary app providing doctors with more detailed information on the patient's activity and diet) or because a purpose to support a healthy lifestyle is 'indirectly medical'. This reference could have also created a foundation to subject the mHealth apps for 'domestic use' to the MDR safety regime. At the same time, while solving one problem it could have created another, namely, a slippery slope towards including not only lifestyle and wellbeing apps, but also generic software into the material scope of the MDR.

Clearly, the legislator still deems necessary to maintain a distinction between 'software [...] specifically intended by the manufacturer to be used for [...] medical purposes' (Recital 18a MDR), and subject to the MDR, and software for general purposes, even used in a healthcare setting, e.g. lifestyle and well-being applications. The latter are not considered medical devices, and as such are not to be subjected to the same safety and performance regime as the medical devices are. ${ }^{50}$ Yet, a significant diversion of the MDR from the MDD is that the Regulation will also apply to 'the groups of products without an intended medical purpose that are listed in Annex XV' (Article 1(1)(a) MDR). The rationale behind this change is the intent of the legislator to subject to safety and performance requirements '[c]ertain groups of products for which the manufacturer claims only an aesthetic or another non-medical purpose but which are similar to medical devices in terms of functioning and risks profile' (Recital 11 M DR). Examples of Annex XV devices are contact lenses or other articles intended to be introduced into or onto the eye, some equipment used in cosmetic procedures such as equipment for liposuction, lipolysis or lipoplasty, and intense pulsed light equipment, etc.

So far Annex XV does not include software or specifically mHealth apps. However, the Commission may add other groups of products, such as wellbeing and lifestyle apps, to the list by way of delegated legislation (Article 1(1)(c) MDR) when such apps appear similar to medical devices in terms of functioning and risks. A risk profile of a lifestyle app is likely to be deemed similar to medical devices when that app produced real health effects and hence creates real health risks in case it does not or mal-functions. It is possible that

\footnotetext{
parl.europa.eu/sides $/$ getDoc.do $?$ type $=$ TA\&language $=\mathrm{EN} \&$ reference $=\mathrm{P}$-TA-2014-0266 (last accessed 5 April 2016), Amendments 65 and 66. 
the similarity to medical devices in functioning refers to the situations where a device is not intended by a manufacturer as a medical device (e.g. an app is marketed as an app for consumer wellbeing use only) whereas the device's actual functions, subjective intent of the manufacturer set aside, are similar to the ones a medical device performs. This is another element pointing towards objectivisation of the MDR compared to the MDD that can bridge the gap in safety and performance regulation of the mHealth lifestyle and wellbeing apps with real health effects.

Since the regime of medical devices (currently, under the MDD and in future under the MDR) is the only safety and performance regime that clearly applies to some categories of health-related software, and more specifically mHealth apps, further analysis will focus on this regime.

\section{$4 \quad$ Legal Status of the eHealth PaaS Components and Providers}

Even in cases where an app unequivocally falls in the material scope of the MDD (or MDR), some inherent features of the eHealth PaaS may create difficulties in legal compliance for the manufacturers of such medical apps using the PaaS 'spare parts' for app development. Namely, the idea of eHealth PaaS is an expression of the modular approach to building apps out of spare parts provided and controlled by third parties. While these generic spare parts may be vital for the safety and performance of a medical app, safety and performance requirements both under the MDD and MDR are unlikely to apply to them, or bind the eHealth PaaS provider with any obligations.

Before demonstrating these points, it is important to note that two scenarios of deployment of eHealth PaaS are possible: First, a generic component available via eHealth PaaS may in itself be 'self-sufficient' stand-alone software which meets all the elements of the definition of a medical device, e.g. modules intended to generate alarms. However, this scenario is less representative of the innovative idea of a PaaS as a tool-box of spare parts for new devices. Second, more consistent with the idea of an eHealth toolbox, eHealth PaaS may provide software that is not self-sufficient as a medical device with its own function, but serves as one of the building blocks for new eHealth devices. This scenario will be the focus of further analysis.

\subsection{Current Legal Status of eHealth PaaS Components and Providers}

Currently, only medical devices and their accessories are subject to the MDD. This section will show that the eHealth PaaS components, when the eHealth 
PaaS operates as a toolbox, providing pre-made generic spare parts for future mHealth apps, cannot be considered a medical device, and can be considered an accessory only under very limited circumstances.

Only stand-alone software with a medical function listed in the definition of a medical device may be qualified as a medical device, 'stand-alone' defined as 'software which is not incorporated in a medical device at the time of its placing on the market or its making available'.51 Some software available via eHealth PaaS may fit into this category and hence may be seen as medical device. At the same time, other components may not have own medical function (e.g. connectivity, data security, etc.), and yet be a critical part of a new eHealth device that combines generic functionalities of the components and produces a new medical functionality. A clear example of this is a data analytics component that is generic but, once built into a medical app, obtains a medical use. When included in a newly built app, the proper functioning of the data analytics component will be critical for the safety and performance of the entire app. Currently, the existing requirements refer to the end product (e.g. the app) and not to the components that are not self-sufficient and have to be incorporated in a device to be operational. Hence, generally, eHealth PaaS and PaaS components are not subject to any MDD requirements.

An exception from this conclusion is the case of eHealth PaaS components qualified as 'accessories' to a medical device. An 'accessory' is:

an article which whilst not being a device is intended specifically by its manufacturer to be used together with a device to enable it to be used in accordance with the use of the device intended by the manufacturer of the device. ${ }^{52}$

This definition requires that there is an intent of the manufacturer of the accessory in question to design it for a specific medical device. It is certainly possible that components specifically designed for a particular eHealth device are made available and powered via a PaaS. However, this form of delivery of eHealth PaaS components defeats the very purpose of the eHealth PaaS - to produce and make available a set of components with generic, i.e. not tailored for a specific device, functionalities that can be combined to build new things. Such PaaS components will most likely not be accessories to medical devices, or under very limited circumstances.

\footnotetext{
$51 \quad$ Supra note $19,6$.

52 Article 1(2)(b) MDD.
} 
A consequence of the eHealth PaaS components not being medical devices under the MDD, the platform and components providers are not considered 'device manufacturers', and are not subject to any obligations to ensure that the components are safe and perform as intended. This is important since the entire MDD regime is effectuated by the obligations that the Directive imposes on the manufacturers..$^{53}$

A statutory obligation to ensure that the device is safe and performs as intended, backed up by a possibility of sanctions, lies with the manufacturer alone. The manufacturer is defined as 'the natural or legal person with responsibility for the design, manufacture, packaging and labelling of a device before it is placed on the market under his own name, regardless of whether these operations are carried out by that person himself or on his behalf by a third party' (Article 1(2)(f) MDD). Device manufacturer under the MDD is not necessarily the one who actually makes the device (e.g., an app developer in case of mHealth). The decisive criterion is the name under which the device is placed on the European market. Any third parties involved in the device production, e.g. contractors, suppliers of spare parts, engineers, and scientists, currently bear no statutory obligations under MDD. Any responsibility for safety and performance of a device on their part will be derived from their contractual relationship with the manufacturer. ${ }^{54}$

Translated to the context of mHealth apps built using the eHealth PaaS 'toolbox', this means the following. Given that the components are not selfsufficient medical devices, and accessories only in limited circumstances, the burden of ensuring safety and performance of the medical apps built with these components (and legal compliance) is for the manufacturers of such medical apps alone. As Section 5 will show, ensuring safety and performance of the medical apps in this multi-actor and multi-component context may prove challenging.

\subsection{Legal Status of eHealth PaaS Components and Providers and the Reform}

The Reform does not change the approach of the regulator to grant the status of a medical device to the final product only, and not to the pre-made generic components, or to grant the status of an accessory when software is intended

53 Alternatively, the obligations are imposed on an authorized representative, in case the manufacturer is not established in the EU.

54 E.g. the manufacturer may include essential requirements among specifications for the device to be developed in the contract with the involved third parties. 
for a specific medical device. ${ }^{55}$ The change of most relevance for the modular approach to building mHealth solutions is the extension of the material and personal scope of the Regulation to include parts and components to medical devices, and their manufacturers. This section will examine if and how this change will affect the status of the eHealth PaaS components and providers.

The MDR seems to recognize the role other economic operators beyond manufacturers and their authorized representatives play in ensuring that only safe medical devices come onto the European market. The MDR extended the range of actors bearing obligations to include importers, distributors and persons who put on the market systems and procedure packs. ${ }^{56}$ In addition, and most importantly for the modular eHealth solutions, the MDR also imposes some responsibilities on the providers of parts and components (Article $21(1) \mathrm{MDR})$. These responsibilities vary according to the type of parts and components.

Article 21 MDR distinguishes two types of the parts and components, this distinction being of consequence for the legal status of those parts and components and of the parties bringing them to the European market. The first group are articles meant to replace similar defective parts in existing devices without changing their intended purpose ('intended specifically to replace an identical or similar integral part or component of a device that is defective or worn in order to maintain or re-establish the function of the device' (Article 21(1) MDR). The parties bringing those articles to the market have an obligation to ensure that the parts and components do not adversely affect the safety and performance of the device, and keepevidence to be presented to the competent authorities. The second group are articles intended specifically to replace a part of a device that significantly change the performance, safety characteristics or an intended purpose of the device. These parts and components are considered medical devices on their own (Article 21(2) MDR),

55 'Accessory to a medical device' is 'an article which, whilst not being a medical device, is intended by its manufacturer to be used together with one or several particular medical device(s) to specifically enable or assist the device(s) to be used in accordance with its/ their intended purpose(s) or to specifically and directly assist the medical functionality of the medical device(s) in view of its/their intended purpose(s)'. (Article 2(1)(2) MDR).

$5^{6}$ Importer has an obligation to verify if the manufacturer complied with his obligations (Article $11 \mathrm{MDR}$ ) and distributor has an obligation to act with due care in relation to the applicable safety and performance requirements and to verify if the manufacturer complied with his obligations Where a distributor considers or has reason to believe that a device is not in conformity with the MDR requirements, 'he shall not make the device available on the market until it has been brought into conformity' (Article $12 \mathrm{MDR}$ ). Providers of systems and procedure packs may bear the obligations of the device manufacturers (Article $20 \mathrm{MDR}$ ). 
and hence are fully subject to safety and performance requirements. The parties bringing them to the market are considered manufacturers, and bear all respective obligations.

eHealth PaaS components could be both, or none of the two, depending on how 'intended specifically' in Article 21(1) and (2) will be interpreted. Interpretation that requires a component to be intended for a specific (type of a) device is more likely interpretation, among others, due to the internal consistency of the provisions of Article 21. More precisely, one may argue that it is impossible to ensure that a component does not compromise a device (Article $21(1)$ ) or establish if the component 'significantly changes the performance or safety characteristics of the device' (Article 21(2)), or change an intended purpose of a device, without knowing what exact (type of a) device is involved. Therefore, it is unlikely that components available via PaaS and meant to be generic, 'mixed and matched' to produce a wide variety of mHealth apps will be within the scope of Article $21 \mathrm{MDR}$, and hence under the MDR regime.

5 Challenges to Safety and Performance of eHealth Devices Built on
eHealth PaaS: Perspective of the Manufacturer's Compliance

Both the MDD and MDR safety and performance requirements were adopted under the so-called 'New Approach'. ${ }^{57}$ The New Approach changed the way how the requirements to goods are harmonised by introducing a distinction between 'essential safety requirements' formulated in directives and mandatory for the distribution of products on European market, and the 'harmonised standards', detailing how the general essential requirements are to be complied with. ${ }^{58}$

Medical software is safe and performs as intended when it complies with the essential requirements to safety and performance of medical devices formulated in Annex I MDD. Under the MDR, these are 'general safety and performance requirements' (Annex I MDR). The requirements differ depending on the nature and intended purpose of a device, but those with direct eHealth relevance can be grouped into three categories:

57 J.-P. Galland, 'The difficulties of Regulating Markets and Risks in Europe through Notified Bodies', Eur. J. Risk Reg. (3) (2013) 367.

$5^{8}$ Council Resolution 'On a new approach to technical harmonization and standards', 85/ C 136/01 7 May 1985, available at http://eur-lex.europa.eu/legal-content/EN/TXT/PDF/?ur $\mathrm{i}=$ CELEX:31985Y0604(01)\&from=EN. 
a. Combination of the components of the eHealth medical device must be safe and perform as intended in its entirety. The entire modular structure of PaaS, plus medical app combination, including the connection systems, must be safe and perform as intended and not impair performance and safety of a medical app (e.g. Section 9.1 Annex I MDD; Section 9.1 Annex I MDR);

b. An eHealth medical device built of the eHealth PaaS components must comply with the state-of-the-art, e.g. as expressed in standards. The software as (or in) a medical device, including apps, must be validated according to the state of art, taking into account the principles of development lifecycle, risk management, validation and verification (Section 12.1a Annex I MDD; Section 14 Annex I MDR). The conformity with this requirement is presumed, if medical software conforms to harmonised standards;

c. Risks that the eHealth device may present have to be mitigated, and residual risks acceptable. The design choices must be made with the following goals:

- eliminate or reduce risks as far as possible;

- in relation to risks that cannot be eliminated, take adequate protection measures including alarms;

- inform users of the residual risks due to any shortcomings of the protection measures adopted;

- any undesirable side-effect must constitute an acceptable risk when weighed against the performances intended (section 6 Annex I MDD; Section I (1a) Annex I MDR).

These three categories of requirements present most compliance difficulties since the eHealth device manufacturers who are legally under the obligation to ensure safety and performance are removed from control over the cloud-based device components, whereas the PaaS providers controlling the components are under no legal obligation to ensure safety and performance under the current law, and it is unlikely that the Reform imposes such obligations.

The challenges that the eHealth device manufacturers face depend on the deployment mode of the PaaS. ${ }^{59}$ Where eHealth PaaS is used to build and run apps as medical devices, the safety and performance requirements

59 U.s. Department of Commerce, National Institute of Standards and Technology, 'The NIST definition of cloud computing. Recommendations of NIST', September 2011, 3 (hereinafter: 'The NIST Recommendations'). 
and obligations of the manufacturers are likely to be 'transposed' onto the platform providers during negotiations and a platform selection process: ensure safety and performance of the application in combination with the underlying infrastructure; meet industry standards, and engage in the risk mitigation. Using an eHealth PaaS via a public cloud creates serious compliance challenges for app developers in their capacity as manufacturers of medical devices, mostly, because of the high degree of dependence on the cloud provider, dynamic but often non-transparent nature of a cloud, and inflexibility of the public cloud infrastructure and terms and conditions that do not necessarily accommodate specific compliance needs.

In a community cloud it may be easier for a group of similar clients (e.g. medical app developers) to negotiate a better compliance strategy with the platform provider. However, some app-specific compliance needs may not be met equally well for all members of the community within one platform. Although PaaS providers are not directly under any legal obligations stemming from the requirements for medical devices, accommodating these in the terms of service may constitute a competitive advantage (e.g. provisions on compliance with relevant standards, evidence of compliance, possibility of audit and the risk management and risk mitigation).

When an eHealth PaaS is offered via a private cloud, it is easier to comply with the legal obligations including compliance with standards and risk mitigation, since the developer is not or minimally dependent on a cloud provider.

eHealth PaaS providers who target medical app developers as their clients will profit from accounting for the safety and performance, relevant standards and risk mitigation requirements for medical devices in their infrastructure, services, policies and procedures. At the same time, deploying eHealth PaaS via community and public clouds is not ruled out, but the costs of transposing the requirements onto these multi-tenant clouds are likely to be higher.

\section{Conclusions}

This article examined the compatibility of the idea of an eHealth PaaS, a cloud-based toolkit for easy building of new health apps out of generic spare parts, with the EU safety and performance requirements to health technology under the current and new regimes. The article started with identifying legal regimes of safety and performance potentially relevant for eHealth PaaS. Despite a shared assumption that safety and reliable performance are vital for trust in any health technology, the applicability of any safety and performance regime to mHealth is still a grey area ridden with legal uncertainty. A broad category of the so-called lifestyle and wellbeing mHealth apps for consumer 
or 'domestic' use are out of the scope of any safety and performance regime: pending a clarification of the concept of product, it is unclear if any software, apps included, falls under a definition of a product and hence within a material scope of the general GPSD; further, no software falls within the scope of the specific regime of MDD when it is not meant by the manufacturer specifically for 'medical use'. This approach to defining a medical device and the scope of the MDD is dependant on the manufacturer's subjective intent. While the Reform operated with largely unchanged definition of a medical device, including the subjective manufacturer's intent, a clear shift towards 'objectivisation' of the regime of safety and performance under the MDR is observable: the application of the safety requirements is triggered also by objective risks to health and a device's functionality similar to the one of a medical device. Further, the Commission will be able to edit a list of the non-medical devices subject to the MDR. mHealth apps are not on this list yet. I expect that, as the non-medical mHealth apps evolve and rely on more sophisticated technology, e.g. Artificial Intelligence, their real health effects could become more obvious and easily demonstrable. This will make the inclusion of the mHealth in the list more likely. Yet, so far only explicitly medical apps are subject to any safety and performance regime, i.e. the regime of medical devices. Therefore, the analysis of how the mapped legal rules would work out in the circumstances of eHealth PaaS focused on the MDD and the MDR.

Both current and future safety and performance regimes generally do not apply to software generic 'spare parts' without a self-sufficient medical function. The MDR does create some obligations for the providers of parts and components, but only when they are specifically meant to replace a worn-off or defective element of the specific medical device, or significantly change the characteristics of a specific medical device. While the eHealth PaaS components meant for a specific (type of) medical app are certainly possible, most components available via PaaS are intended to be generic, 'mixed and matched' to produce a variety of mHealth apps, and not the apps of a particular type or functionality. Therefore, most PaaS components are not likely to fall within the scope the MDR. The Reform, while introduces some requirements for the 'spare parts', will have limited effect in the particular circumstances of the eHealth components offered via PaaS.

The inherent consequence of using eHealth PaaS 'spare parts' to build 'regulated' mHealth apps is a tension between the ease of building and running apps out of pre-made spare parts where no own expertise and IT capacity is needed, on the one hand, and compliance by the manufacturers of the finished apps with the obligations to ensure safety and performance of these apps on the other hand. The mHealth device manufacturers are legally under the obligation to ensure that their app is safe in use and in combination with 
other devices, performs as intended, and does not present unacceptable risks. This is challenging when the manufacturer is effectively removed from control over the cloud-based app components, while the PaaS component providers in control of the components are under no legal obligation to ensure safety and performance under the current law. The Reform will not change the situation significantly.

This tension may be resolved in part by configuring how the eHealth PaaS is deployed. Generally, the heavier the regulatory burden in a sector is, the more likely it is that the choice of a private or community cloud is better from the compliance perspective. This is because community and private clouds, unlike public clouds, allow clients more control over the cloud infrastructure, processes and policies to ensure, verify and be able to demonstrate legal compliance.

Where the platform is used to build eHealth solutions that are not intended as medical devices, there are no clear safety and performance related restrictions for deployment of the eHealth PaaS. This is true regardless of whether or not such apps are meant for a medical or consumer context. At the same time, to provide equal protection for consumer health some form of safety and performance requirements and restrictions may apply to lifestyle and wellbeing apps and respective eHealth PaaSs in future: if the material scope of the GPSD is interpreted to include software, or the Commission includes mHealth apps with health effects into the list of non-medical products similar to medical devices in their functionality or risks. Although at present it is not yet clear if and to what extent lifestyle and wellbeing apps pose a risk to health, ${ }^{60}$ the risks may become more apparent as the range of application and functionalities of such eHealth platforms grow, and the apps use e.g. persuasive technologies or influence health-relevant behaviour of their users in another way. Such regulatory changes will change the legal landscape of the eHealth PaaS and its preferred deployment options.

At present, however, the main 'selling features' of the eHealth PaaS, namely, outsourced expertise and burden of creating and maintaining cloud-based 'spare parts' for mHealth 'quick fixes', do not easily fit in the legal landscape of mHealth. Even more so, those selling points present the main obstacles for legal compliance for the manufacturers of the regulated medical apps. The Reform will not change this significantly. Market may push PaaS providers to offer medical mHealth manufacturers tools to ensure and control safety and performance. I foresee no such incentives in the yet unregulated field of consumer mHealth. 